\title{
Evolution of Flow Cytometry Technology
}

\section{Guilherme F Silveira*}

Laboratório de Virologia Molecular, Instituto Carlos Chagas, Curitiba, Brasil

Keywords: Flow cytometry; Cell characterization

\section{History}

In 1953, the American electric engineer, Wallace H Coulter, developed a system for counting cells. Based on the Coulter principle, which had been discovered in the 1940s, this device determined the size of cells in a flowing solution based on the impedance principle. The size of a particle is determined by the transient proportional current measured by electrodes as the particle is pulled through them. The change in impedance is transient and proportional to the size of the particle (i.e., a virus or a eukaryotic or prokaryotic cell) tested. This mechanism formed the foundation of the technology that we use to determine cellular parameters and enabled the collection of quantitative and qualitative observations of a living organism's biomarkers. Building on the Coulter principle (Figure 1A), Mack Fulwyler invented a device for cell separation [1]. This equipment used the difference in cell size to select, with an electrostatic field, drops containing cells of a determined size for collection in a series of vessels. Next, Len Herzenberg coined the term FACS (Fluorescence-activated cell sorting) to describe a cell separation system in which fluorescence is used as a cell sorting mechanism [2]. Although advances have been made in the separation technologies employed by flow cytometry, some of the foundational principles remain in use today, such as the use of electric fields to direct droplets containing cells of interest. The development of new technologies to increase the performance, robustness, resolution and recovery rate in flow cytometric devices led to the creation and development of novel equipment. In 1968, Wolfgang H Göhde, in collaboration with Wolfgang M Dittrich, developed a flow cytometry system that sorted by intracellular fluorescence [3]. Göhde's major contribution to the field of flow cytometry was the creation of the flow chamber, which remains in use today. With this chamber, it is possible to form a focal point that allows the particles, or cells, to be analyzed individually and more efficiently than in previous devices. In Göhde's original work, analyses are performed using optical microscopes, which were later replaced by polarized light detectors (i.e., lasers). This enabled the increased response capacity of the equipment. Another of Göhde's contributions was the development of methods based on photon emission by fluorescent molecules rather than absorption, which had been more widespread at the time. In subsequent decades, several companies developed commercial equipment that ushered in a new era in the analysis of cells. Equipment, such as, the Cytofluorograph (1971) from Bio/Physics Systems Inc., the PAS 8000 (1973) from Partec, the first FACS instrument from Becton Dickinson (1974), the ICP 22 (1975) from Partec/Phywe and the Epics from Coulter (1978). Currently, the most prevalent hematological flow cytometry equipment is produced by the Coulter Company; this high performance equipment is used both for research and clinical analysis. The largest manufacturer of equipment used in basic and applied research is Becton Dickinson (BD), with cytometers that are endowed with several different lasers and additional features. The equipment currently in use has considerable advantages over earlier technology (Figure 1). Early flow cell readers were based on the electrostatic properties of the samples, but today, they detect fluorescence. The determination of both the volume and complexity of samples was also limited in early equipment. Today, the evolution of systems integrating fluidics, optics and electronics allows the determination of multiple parameters in a complex population. Therefore, to understand the evolution of flow cytometry technology, it is necessary to become familiar with the technology utilized in current equipment.

\section{Current Technology}

Current flow cytometry equipment contains three basic component systems: fluidics, optics and electronics [4]. In cytometry systems, the fluid systems comprise the pump, pipelines, valves and chambers that are used for pressurizing, organizing and directing the buffer containing the cells. The fluid system arranges the samples by hydrodynamic focusing tailored for different flow characteristics. Once organized, the cells are directed to the interrogation point in the flow chamber, where they are intercepted by one or more lasers. After this point, cells can be separated and recovered within the cell-sorter equipment or discarded, depending on the equipment characteristics and/or experimental need. The interrogation point is the intersection between the fluid system and the optical system. In general, flow cytometry equipment uses diode lasers, lamps (typically mercury or xenon), or elements such as argon, $\mathrm{HeNe}$, and $\mathrm{HeCd}$ as the emission source. The lens and prism system focuses these laser beams at the interrogation point where the laser intersects with the cells or particles. This laser incidence results in refraction and/or diffraction of a light beam. The sample size and complexity of features are directly related to the amount of redirected light. Flow cytometers use photodetectors or photomultipliers to detect the laser; light refracted at small angles $\left(<30^{\circ}\right)$ is correlated with cell size, while light refracted at larger angles $\left(>30^{\circ}\right)$ is correlated with the internal complexity of the cells. Additionally, the lasers excite fluorescent molecules. One of the advantages of current flow cytometry is the ability to analyze multiple factors present in cells or particles in suspension at a high resolution. To perform these multiparametric analyses, photoexcitable chemical compounds, proteins, or nanomaterials can be employed. These fluorescent agents (or chromogens) can be conjugated to antibodies (for recognition marker proteins) or can be dyes delivered directly into cells as markers of macromolecules such as DNA, lipids or proteins, or as byproducts of

*Corresponding author: Guilherme F Silveira, 1-Laboratório de Virologia Molecular, Instituto Carlos Chagas, Curitiba, Brasil, Tel: +55(41)3316-3230; E-mail: gfsilveira@gmail.com

Received July 29, 2015; Accepted August 25, 2015; Published August 31, 2015 Citation: Silveira GF (2015) Evolution of Flow Cytometry Technology. J Microb Biochem Technol 7:4 213-216. doi:10.4172/1948-5948.1000208

Copyright: (C) 2015 Silveira GF. This is an open-access article distributed under the terms of the Creative Commons Attribution License, which permits unrestricted use, distribution, and reproduction in any medium, provided the original author and source are credited. 
A

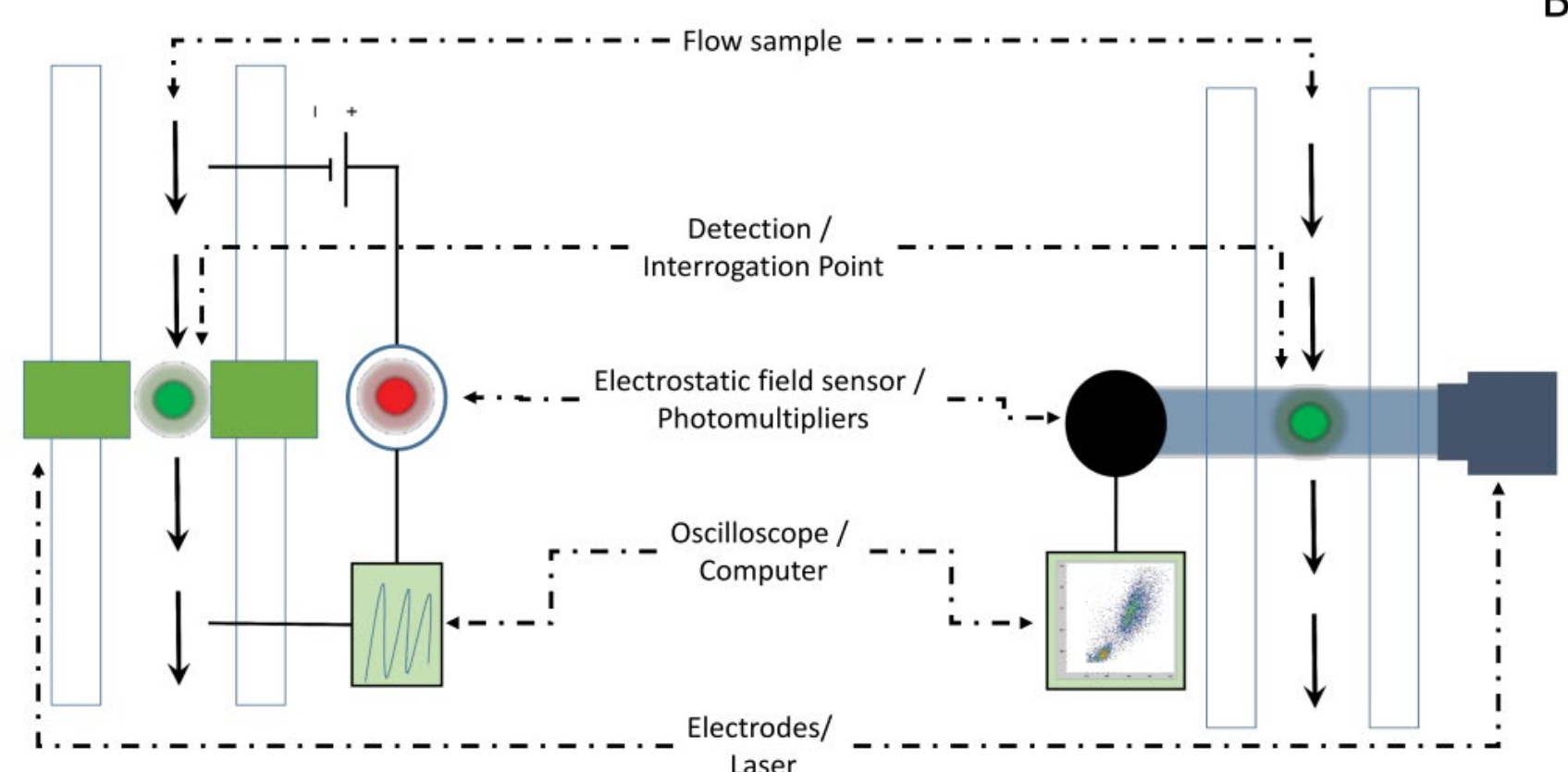

Figure 1: Comparisons between early flow cytometry technology and current systems. Several modifications have been included to illustrate the significant increase in system complexity between early (A) and current flow cytometers (B).

other chemical interactions. Regardless of the form of the chromogen, flow cytometry can be used to detect the light signal emitted by the compound at the laser interrogation point. This light is filtered, and specific emission wavelengths are detected by a photomultiplier. This captured light is then converted into electronic signals that are translated as mathematical values representing fluorescence intensity by the electronics system of the flow cytometer. The electronics system is therefore responsible for capturing the light signal and converting it into electrical pulses (similar to those described by the Coulter principle in early flow cytometry). This electronic signal gives rise to an array of dimensionless values related to the intensity of the light emitted, which are made available to the user in dot plot or histogram format. Using these graphs, the user can visualize the layout of average fluorescence intensity (MFI) or the percentage of cells (or particles) positive and negative for each marker [4]. Using the analysis strategies of exclusion and inclusion population gates, the user may determine the parameters of interest for the analysis of flow cytometry data.

\section{Evolution of Flow Cytometry}

Several important developments in flow cytometry technology are in use today. The use of more robust photodetectors and new laser emitters, the use of LED lamps as emitter, and changes in analytical capacity (multidimensional analysis software) are examples of recent improvements. Although these represent important advances, a major overhaul in flow cytometry consists of new approaches combining existing technologies with routine laboratory procedures, such as imaging. This makes possible the acquisition of images of cells in real time as they pass by the interrogation point. At this point, the presence of target markers is assessed, and the subcellular localization of these markers can be imaged. Although imaging during cytometry has long been proposed [5], it is not yet widely used by research laboratories, probably due to the low image resolution. However, over the last 10 years, technology has advanced to the point where it is now possible to resolve some of the issues with this approach. Another technology that is already a reality but that is not yet widespread is flow cytometry accompanied by mass spectrometry (by time-of-flight, or TOF). Relatively recently [6], the mass cytometry approach has been characterized by coupling with a fluid flow cytometry system, which allows the separation and organization of cells in suspension with the resolution of mass spectrometry. Using antibodies labeled with nonfluorescent heavy metals, cells are separated one-by-one and injected into an ionization chamber, where the metals bound to antibodies are identified by their TOF characteristics. This is not yet a widely used technology, but some researchers believe it will be possible to simultaneously assay up to 100 markers in each cell by mass cytometry.

\section{Flow Cytometry Application and Analysis}

Flow cytometry has led to great advances in cytology, hematology, immunology, and microbiology, among other research fields. The qualitative and quantitative assessment of pathogens, cell response phenotype and cell death, as well as the study of metabolic functions, cell-cell and cell-extracellular matrix interactions, are made possible 
using flow cytometry techniques. Publications in the fields of hematology [7], immunology [8,9], pathology [10], calcium processing [11], immunophenotyping [12], and others [13-15] highlight the breadth of cytometry applications. In addition to clinical applications, fields such as food science [16], environmental science [17], botany $[18,19]$, aquatic microbiology [20], veterinary science [21], and physics [22] have benefited from this technology. This shows the broad reach of flow cytometry techniques, which can assist with data collection in many different fields. These flow cytometry data are generated by specific fluorescence markers and can be analyzed in two ways: by obtaining either the percentage or the MFI of cells for the markers of interest. The data types are correlated and are not redundant. The determination of the percentage of cells enables the separation of positive and negative populations that express a marker of interest in response to a treatment. This percentage may be further correlated with morphological changes (i.e., the size and complexity of cells) and other markers of interest. The number of markers used directly influences this correlation. In a simple experimental model, flow cytometry data obtained for three specific markers yielded 40 different correlations in each of the cells acquired during data collection. Additionally, determining the MFI allows the observation of different concentrations of a marker present in the target cells. For example, a particular protein may be overexpressed under certain experimental conditions and even under control conditions. These characteristics demonstrate that with a relatively low investment, it is possible to obtain detailed data using current flow cytometry technology. The flow cytometry technology available today includes different devices with several configurations. Commercially available equipment can now be equipped with anywhere from one laser, which can determine five parameters, to 5 lasers, which can determine up to 20 parameters simultaneously. Additionally, flow cytometry technology is evolving to enable the acquisition of a greater number of parameters in the same sample.

\section{Conclusion}

Flow cytometry has evolved from its inception by Coulter in the 1950s to the indispensable multiparametric analysis platforms in use today. As described in this review, many options are available today, including different laser configurations and many photodetectors. The use of antibodies and other markers to label different cellular characteristics is now a reality and allows a thorough analysis of cellular systems. These parameters can be analyzed under different conditions (i.e., MFI and/or percentage) and can be correlated with the biological and/or biochemical changes in the samples. Examples of how flow cytometry has enhanced the understanding of complex systems abound. Additionally, flow cytometry is a frequent target of technological (i.e., image flow cytometry and CyTOF) methodological developments. Today the application of flow cytometry is limited only by the researcher's creativity.

\section{References}

1. Fulwyler MJ (1965) Electronic separation of biological cells by volume. Science 150: $910-911$.

2. Julius MH, Masuda T, Herzenberg LA (1972) Demonstration that antigenbinding cells are precursors of antibody-producing cells after purification with a fluorescence-activated cell sorter. Proceedings of the National Academy of Sciences of the United States of America 69:1934-1938.

3. Dittrich WM, GAhde WH (1968) Flow-through Chamber for Photometers to Measure and Count Particles in a Dispersion Medium. United States Patent 3: $76-187$.

4. Becton, Dickinson (2000) Introduction to Flow Cytometry: A Learning Guide. 1: 54 .

5. Kachel V, Benker G, Lichtnau K, Valet G, Glossner E (1979) Fast imaging in flow: A means of combining flow-cytometry and image analysis. J Histochem Cytochem 27: 335-341.

6. Bandura DR, Baranov VI, Ornatsky OI, Antonov A, Kinach R, et al. (2009) Mass cytometry: Technique for real time single cell multi-target immunoassay based on inductively coupled plasma time-of-flight mass spectrometry. Anal Chem 81: 6813-6822.

7. De Rosa SC, Herzenberg LA, Herzenberg LA, Roederer M (2001) 11-color, 13-parameter flow cytometry: identification of human naive $T$ cells by phenotype, function, and T-cell receptor diversity. Nat Med 7: 245-248.

8. Rothe G, Schmitz G (1996) Consensus protocol for the flow cytometric immunophenotyping of hematopoietic malignancies. Working Group on Flow Cytometry and Image Analysis. Leukemia 10: 877-895.

9. Loken MR, Stall AM (1982) Flow cytometry as an analytical and preparative tool in immunology. Journal of immunological methods 50: R85-R112.

10. Willman CL, Stewart CC (1989) General principles of multi-parameter flow cytometric analysis: Applications of flow cytometry in the diagnostic pathology laboratory. Semin Diagn Pathol 6: 3-12.

11. Barten MJ, Gummert JF, van Gelder T, Shorthouse R, Morris RE (2001) Flow cytometric quantitation of calcium-dependent and -independent mitogenstimulation of $\mathrm{T}$ cell functions in whole blood: inhibition by immunosuppressive drugs in vitro. Journal of immunological methods 253: 95-112.

12. Schmid I, Uittenbogaart CH, Keld B, Giorgi JV (1994) A rapid method for measuring apoptosis and dual-color immunofluorescence by single laser flow cytometry. J Immunol Methods 170: 145-157.

13. Guindulain T, Comas J, Vives-Rego J (1997) Use of nucleic acid dyes SYTO13, TOTO-, and YOYO-1 in the study of Escherichia coli and marine prokaryotic populations by flow cytometry. Appl Environ Microbiol 63: 4608-4611.

14. Davey HM, Kell DB (1996) Flow cytometry and cell sorting of heterogeneous microbial populations: The importance of single-cell analyses. Microbiol Rev 60: 641-696.

15. Fouchet P, Jayat C, Héchard Y, Ratinaud MH, Frelat G (1993) Recent advances of flow cytometry in fundamental and applied microbiology. Biol Cell 78: 95-109.

16. Comas-Riu J, Rius N (2009) Flow cytometry applications in the food industry. J Ind Microbiol Biotechnol 36: 999-1011.

17. Liu B, Liang H, Qu F, Chang H, Shao S, et al. (2015) Comparison of evaluation methods for Microcystis cell breakage based on dissolved organic carbon release, potassium release and flow cytometry. Chemical Engineering Journal 281: 9.

18. Dolezel J, Greilhuber J, Suda J (2007) Estimation of nuclear DNA content in plants using flow cytometry. Nat Protoc 2: 2233-2244.

19. Suda J, Travnicek $P$ (2006) Reliable DNA ploidy determination in dehydrated tissues of vascular plants by DAPI flow cytometry--new prospects for plant research. Cytometry Part A: The journal of the International Society for Analytical Cytology 69:273-280.

20. Vives-Rego J, Lebaron P, Nebe-von Caron G (2000) Current and future applications of flow cytometry in aquatic microbiology. FEMS Microbiol Rev 24: 429-448. 
21. Davis EG, Wilkerson MJ, Rush BR (2002) Flow cytometry: clinical applications in equine medicine. J Vet Intern Med 16: 404-410.
22. Oakey J, Applegate RW Jr, Arellano E, Di Carlo D, Graves SW, et al. (2010) Particle focusing in staged inertial microfluidic devices for flow cytometry. Anal Chem 82: 3862-3867. 